\title{
Microarchitectures et architecture gothique
}

Notes de lectures juxtaposées : Microarchitectures médiévales. L'échelle à l'épreuve de la matière, Jean-Marie Guillouët et Ambre Villain (dir.), Paris, Éditions Picard et Institut national d'histoire de l'art, 2018 ; Qu'estce que l'architecture gothique ? Essais, Arnaud Timbert (dir.), Villeneuve-d'Ascq, Presses universitaires du Septentrion, 2018.

\section{Claire Pernuit}

\section{(2) OpenEdition}

Édition électronique

URL : https://journals.openedition.org/cem/17326

DOI : $10.4000 /$ cem.17326

ISSN : 1954-3093

Éditeur

Centre d'études médiévales Saint-Germain d'Auxerre

Référence électronique

Claire Pernuit, " Microarchitectures et architecture gothique », Bulletin du centre d'études médiévales d'Auxerre | BUCEMA [En ligne], 24.1 | 2020, mis en ligne le 21 septembre 2020, consulté le 22 septembre 2022. URL : http://journals.openedition.org/cem/17326 ; DOI : https://doi.org/10.4000/ cem. 17326

Ce document a été généré automatiquement le 22 septembre 2022.

\section{(c) (i) 80}

Creative Commons - Attribution - Pas d'Utilisation Commerciale - Partage dans les Mêmes Conditions 4.0 International - CC BY-NC-SA 4.0

https://creativecommons.org/licenses/by-nc-sa/4.0/ 


\section{Microarchitectures et architecture gothique}

Notes de lectures juxtaposées : Microarchitectures médiévales. L'échelle à l'épreuve de la matière, Jean-Marie Guillouët et Ambre Villain (dir.), Paris, Éditions Picard et Institut national d'histoire de l'art, 2018 ; Qu'estce que l'architecture gothique ? Essais, Arnaud Timbert (dir.), Villeneuve-d'Ascq, Presses universitaires du Septentrion, 2018.

\section{Claire Pernuit}

Ces deux ouvrages représentent l'aboutissement de colloques tenus respectivement en 2014 à Paris (INHA) Microarchitectures médiévales - et en 2015 à Chartres (Centre international du vitrail) - Qu'est-ce que l'architecture gothique? À première vue, l'"échelle", pour reprendre l'un des mots-clés du sous-titre du premier, sépare les deux publications : la «micro» architecture, miniature ou miniaturisée, s'opposant au monumental. Dans le contexte des années 1970, sous la plume de François Bucher ${ }^{1}$, cette opposition rationnelle s'était vue adjoindre une dimension plus sociale: au " petit » intime, la proximité avec le fidèle

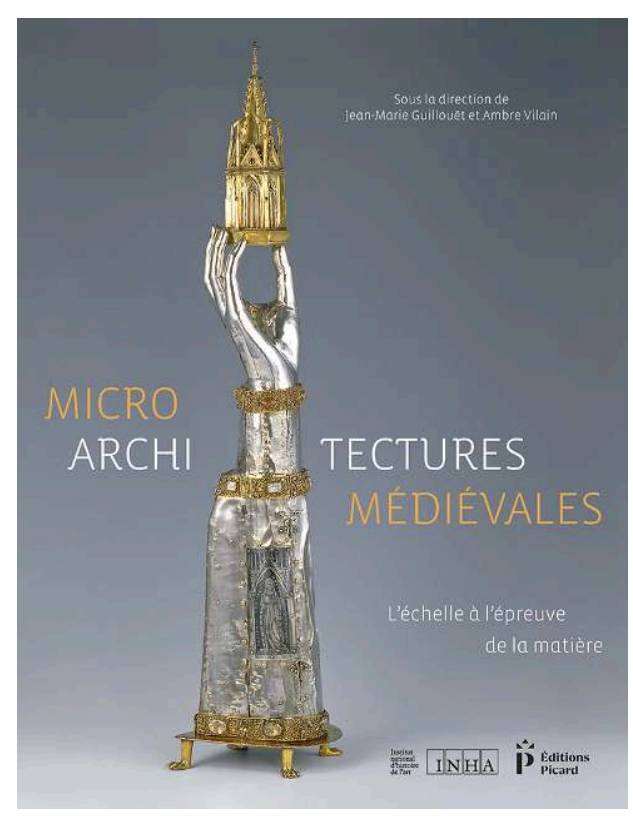
et le pèlerin, tandis que le "grand", intimidant, se faisait le reflet des visions des élites ; l'historiographie des décennies suivantes a progressivement relativisé ces positions tant une même idée a pu être à l'œuvre dans chaque catégorie. Loin de ces problématiques, tout en les gardant en mémoire, ces deux publications se sont surtout concentrées sur la définition et la place de leurs disciplines dans le contexte actuel de la 
recherche française et internationale. Après la présentation juxtaposée de ces deux contributions, nous proposerons quelques remarques d'ensemble.

2 Fruits d'une collaboration des éditions de l'INHA et des éditions A. et J. Picard, les actes du colloque Microarchitectures médiévales, l'échelle à l'épreuve de la matière sont euxmêmes issus de la rencontre de deux projets de recherche, menés pour l'un sur l'architecture " habitée » sous la responsabilité d'Ambre Vilain à l'INHA de 2012 à $2014^{2}$, et pour l'autre sur la miniaturisation dans l'art et l'artisanat sous la coordination de Jean-Marie Guillouët, de 2014 à 2018, à l'université de Nantes³. Cette manifestation, initialement intitulée «Microarchitecture et figures du bâti, l'échelle à l'épreuve de la matière » et tenue à l'INHA du 8 au 10 décembre 2014, avait donc, selon les propos introductifs des deux auteurs, pour ambition de confronter les résultats de ces réflexions concomitantes. Mais, plus encore que la concrétisation de la rencontre de deux thématiques complémentaires, la publication des actes semble avoir été envisagée comme un bilan des problématiques posées par ces représentations miniatures, confirmées comme constituant un domaine de recherche à part entière dans l'histoire de l'art depuis un premier colloque tenu à Nuremberg en 2005'. En témoignent la conversion au pluriel du terme principal du titre - « Microarchitectures médiévales » -, mais également les efforts historiographiques de Jean-Marie Guillouët et de Paul Binski qui constituent la première section (« Principes »).

D'emblée, l'influence de la pensée de François Bucher est prégnante, tant dans cette partie que dans un certain nombre de contributions. La publication en 1976 de « MicroArchitecture as the "Idea" of Gothic Theory and Style ${ }^{5}$ " a en effet constitué, selon la formule de Jean-Marie Guillouët, l'acte de naissance de la microarchitecture comme un « objet scientifique [et] historique ${ }^{6}$ ». Notons que la réédition du même article en 1978, sans modifications majeures, contenait toutefois une orientation nouvelle du titre: alors que la première mouture mettait l'accent sur l'Idea, dans le sens platonicien d'une forme intelligible, la seconde version - "Microarchitecture" as the Idea Of Gothic Theory and Style ${ }^{7} »$ - traduisait l'importance déjà donnée au néologisme inventé par l'auteur et à la nécessité de son adoption pour désigner une typologie d'objets et de représentations. Mais si Jean-Marie Guillouët et Paul Binski saluent conjointement l'impact majeur de cette étude, ils la mettent immédiatement en perspective par la constitution d'un bilan historiographique nécessaire pour le premier et par une relecture contextualisée pour le second. L'associant aux derniers sursauts de la contreculture des années 1960 dans la recherche anglo-saxonne, Paul Binski réfute le discours transcendantaliste au cœur du propos de François Bucher, qui opposait les manifestations grandioses, mais jugées élitistes, de l'architecture monumentale, à l'intimité du lien créé par les espaces réduits des objets sacrés avec les fidèles et pèlerins. L'auteur invite alors à laisser de côté la notion d'échelle des œuvres, secondaire selon lui, tant les manifestations du grand et du petit peuvent relever d'une même idée, et à considérer comme centrale la question de l'artifice et de la technique, autrement dit la "porte d'entrée vers le sublime de l'œuvre ${ }^{8}$ ». En parallèle de cette recommandation stimulante, les contributions introductives sont également l'occasion de rappeler la chronologie et les typologies communément admises, et tout particulièrement le tournant qu'a pu représenter la décennie 1240 dans le foyer 
francilien pour la production miniature, qui sera dès lors durablement imprégnée de l'esthétique des réalisations monumentales contemporaines.

4 La relation entre architecture bâtie et microarchitecture avait déjà largement occupé les débats du colloque de Nuremberg, sans toutefois dépasser les frontières chronologiques et géographiques de la fin du Moyen Âge occidental chrétien. Dans la continuité de ce premier travail et de bien d'autres contributions antérieures - Achim Timmermann ${ }^{9}$, Peter Kurmann ${ }^{10}$-, les différentes communications de cette nouvelle publication ouvrent désormais plus largement la question, dépassant les repères de temps et d'espace à l'instar de l'analyse d'Ethan Matt Kavaler ${ }^{11}$. La problématique de la culture visuelle et architecturale des commanditaires, ainsi que la mise en scène de leur pouvoir, est notamment envisagée à travers un intérêt, sans précédent dans ce contexte, porté à l'étude des sceaux de personnalités laïques et ecclésiastiques, qui ont attiré tout particulièrement l'attention de Julian Gardner ${ }^{12}$, de Matthew James Sillence ${ }^{13}$, ainsi que d'Ambre Vilain et de Clément Blanc-Rieh ${ }^{14}$. Le regard porté sur l'orfèvrerie est également enrichi par l'analyse d'Anita Paolicchi ${ }^{15}$, dans les frontières méconnues de l'ancienne principauté de Valachie (Roumanie), de plusieurs chivote, tabernacles de métal doré conçus comme une reproduction miniature fidèle de l'église dans laquelle ils devaient prendre place. À partir de la constitution d'un corpus de crosses abbatiales et épiscopales, Frédéric Tixier offre également une importante étude de la progressive architecturalisation de ces insignes religieux dans les derniers siècles du Moyen Âge, mêlant analyse technique et portée symbolique du phénomène ${ }^{16}$. La définition la plus complète des objets de la microarchitecture est finalement offerte par Javier Ibáñez Fernández et Arturo Zaragozá Catalán ${ }^{17}$ : « Microarchitecture can refer to objects, representations, and even to entire parts of a construction that aim to totally or partially reproduce, on a small scale, real or imaginary structures. » Soulignant le peu d'études consacrées à la microarchitecture dans l'Espagne de la fin du Moyen Âge (XIV ${ }^{e}-\mathrm{XVI}^{\mathrm{e}}$ siècle), les deux auteurs soumettent également une intéressante proposition de typologie des fonctions des objets de la microarchitecture qui, nonobstant l'interconnexion fréquente de ces différentes catégories, gagnerait à être étendue à d'autres contextes. Un effort comparable de classification a mené Achim Timmermann à distinguer, pour la France du Nord et le Saint Empire romain germanique, trois temps d'attention particulière à ces architectures miniatures, de 1100 aux décennies précédant la Réforme ${ }^{18}$.

5 La présence massive des objets dans l'ouvrage n'est toutefois pas exclusive. Deux contributions explorent plus particulièrement le cas des images des manuscrits : autour de la figure de la Vierge à l'enfant dans le Sherborne Missal ( $\mathrm{xv}^{\mathrm{e}}$ siècle) pour Alexander Collins $^{19}$ et autour de l'affirmation et de l'exaltation visuelle de la souveraineté terrestre carolingienne dans les pleines pages des manuscrits du milieu impérial entre 845 et 875 pour Anne-Orange Poilpré2 ${ }^{20}$ - souveraineté cependant toujours légitimée par la présence de l'autorité ecclésiale, et plus généralement religieuse. Enfin, dans une démarche singulière et quelque peu marginale, Farah Makki propose un examen de l'important corpus scripturaire inscrit sur les murs des palais de l'Alhambra de Grenade et des relations entretenues entre calligraphie et architecture ${ }^{21}$.

6 Le panorama ainsi dressé n'aurait pas été complet sans la prise en compte de deux sujets déjà largement évoqués par l'historiographie: celui de la maquette d'architecture et celui des architectures miniatures encadrant les « images des seuils » des églises ${ }^{22}$. Les différentes lectures de la présence du premier dans les représentations 
de fondateurs aux $\mathrm{xIII}^{\mathrm{e}}$ et $\mathrm{XIV}^{\mathrm{e}}$ siècles - démonstration technique, célébration et perpétuation de l'acte de fondation, entre autres - sont abordées méthodiquement par Sabine Berger avec une attention toute particulière portée au cas original de la collégiale Notre-Dame d'Écouis et à l'ambition unique d'Enguerrand de Marigny et de son épouse, Alips de Mons ${ }^{23}$. À la suite des vœux formulés par l'auteure elle-même en conclusion, on souhaitera la poursuite de telles analyses pour d'autres sites. Quant aux fonctions de la microarchitecture dans la conception des portails gothiques, abordées à la fin de l'ouvrage, elles sont graduellement énumérées et examinées par Sophie CloartPawlak, de l'évident ordonnancement des registres aux principes, plus controversés par la riche bibliographie disponible, de hiérarchisation et de mémorisation ${ }^{24}$.

La richesse des différentes interventions qui constituent cet ouvrage de référence et le travail effectué sur la microarchitecture médiévale ne sont pas sans rappeler le projet comparable mené pour l'architecture gothique sous la direction d'Arnaud Timbert et publié en $2018^{25}$.

Riche de douze contributions soumises à Chartres les 29 et 30 mai 2015, ce volume sur Qu'est-ce que l'architecture gothique? s'inscrit notamment dans la continuité d'un colloque tenu en 2009 à Noyon et objet d'une édition en 2012, également sous la direction d'Arnaud Timbert, alors jointe à celle de Stéphanie-Diane Daussy ${ }^{26}$. Offrant un bilan de l'évolution des méthodes d'analyse de l'architecture et de la sculpture gothique, les auteurs, dont le regretté Andrew Tallon ( $†$ 2018), appelaient alors à un « renouvellement des méthodes et des regards $»^{27}$. À cette première invitation de 2012, répond l'intitulé éloquent - Qu'est-ce que l'architecture gothique? - de ce nouveau recueil.

9 Le titre lui-même mérite attention. L'emploi du singulier n'occulte pas, selon les mots mêmes du texte de présentation, la « multiplicité d'architectures gothiques » qui ont pu apparaître $d u \mathrm{XII}^{\mathrm{e}}$ au XVI $\mathrm{X}^{\mathrm{e}}$ siècle dans des formes et des contextes très divers, sans mentionner les réappropriations tardives de la période moderne. L'interrogation prend en réalité plutôt la forme d'un état des lieux : au-delà des disciplines et sous-disciplines, des différentes périodes et zones géographiques étudiées par chacun, quelle définition donner à l'architecture gothique aujourd'hui ? L'ensemble des communications est du reste représentatif des différentes approches actuelles. Après un bilan historiographique dressé par Arnaud Timbert en introduction ${ }^{28}$, Xavier Barral i Altet fournit une contribution foisonnante construite autour de la cathédrale de Chartres et de l'héritage de Louis Grodecki ${ }^{29}$, rappelant entre autres l'origine du terme gothique, dénomination péjorative dont la recherche se contente toujours aujourd'hui ${ }^{30}$, à défaut d'autres outils linguistiques encore à inventer ${ }^{31}$. Une démarche sémantique et épistémologique semblable est au cœur de la communication de Nicolas Reveyron ${ }^{32}$, appelant à mettre de côté périodisations et classements pour considérer désormais la notion de " polychronie » dans l'émergence et le développement de cette esthétique ${ }^{33}$; les interrogations de Thomas Flum offrent une réflexion complémentaire sur la pertinence d'une période tardive du gothique, parfois étirée sur près de deux cents ans $^{34}$. Le projet de la cathédrale, - «hypostase de l'architecture gothique » selon les 
mots de Christian Freigang ${ }^{35}$-, est ensuite envisagé sous l'angle des commanditaires par Lindy Grant ${ }^{36}$ et Stephen Murray ${ }^{37}$. Le contexte, dans l'ensemble très français de ces différents échanges, est heureusement ouvert à l'expression du gothique européen, dans le contexte des royaumes de Castille et de León aux XII ${ }^{\mathrm{e}}$ et XIII ${ }^{\mathrm{e}}$ siècles par Eduardo Carrero Santamaria ${ }^{38}$ et dans l'ancienne Moravie aux $\mathrm{xIV}^{\mathrm{e}}$ et $\mathrm{xV}^{\mathrm{e}}$ siècles, et tout particulièrement à Prague, par Klára Benešovská ${ }^{39}$. Enfin, la présentation par Nishida Masatsugu du cas des églises "visiblement gothiques" construites par les pères français dans la région de Nagasaki à la fin du $\mathrm{XIX}^{\mathrm{e}}$ siècle ouvre avec intelligence le débat aux « autres idées d'architecture $»^{40}$. Reste toutefois en suspens la question de la définition de l'architecture gothique, à travers et malgré ses expressions diverses. Une certaine gêne est toujours palpable lorsque cette question « justifiée ${ }^{41}$ » est posée et, à défaut d'une réponse universelle, on pourrait avancer, à la suite de Patrice Ceccarini, qu'il ne s'agit pas uniquement d'un "paradigme technologique purement constructif » canonique avec ses arcs-boutants, son élévation à trois ou quatre niveaux, ses voûtes sur croisées d'ogives, ni d'une "pure pensée esthétique ${ }^{42}$, mais que la nature du « liant » entre les deux reste encore à définir.

10 Mais si les bilans ponctuels d'une recherche en évolution sont toujours intéressants dans une certaine mesure, l'ouvrage ne se limite pas à un simple recueil sur l'approche actuelle du sujet. L'omniprésence de la question-titre - Qu'est-ce que l'architecture gothique? - dans chaque contribution pose plus encore la question de l'avenir: en prenant en compte les problématiques d'hier et d'aujourd'hui, comment poursuivre cette étude demain? On serait tenté de répondre: mieux, ou du moins en espérant éviter certains écueils que les différents essais énoncent. Celui de la chronologie et de la datation comme idée directrice doit être considéré comme une priorité, mais c'est également le plus difficile à contourner. Toute l'histoire de l'art du XIx ${ }^{e}$ et du $\mathrm{xx}^{\mathrm{e}}$ siècle a été construite autour d'une chronologie et d'appellations obsolètes - "gothique précoce", "premier gothique», gothique « rayonnant», gothique «tardif». Cette histoire, faite de successions d'édifices toujours plus hauts et toujours plus grands, des premières recherches de Saint-Denis jusqu'à Saint-Pierre de Beauvais et ses désastres, n'est pas sans confort. Mais cette chronique de l'architecture gothique, construite sur le principe d'une naissance, d'un développement, puis d'une progressive agonie, ne saurait être désormais sérieusement adoptée, tout comme la bonne vieille idée du modèle et de la copie. En effet, elles oblitèrent volontairement les spécificités de chaque édifice, le contexte de création de chaque église, entourée de son clergé et son histoire propre. S'attacher à de tels concepts serait encore ignorer la simultanéité des créations, alors que, comme le soulignait à une autre occasion Arnaud Timbert ${ }^{43}$, «il n'échappe en effet à personne que l'architecture gothique n'apparaît pas à Saint-Denis puis à Sens, mais à Saint-Denis et à Sens ${ }^{44}$; et, plus encore, à Saint-Germer-de-Fly (chevet) et à Cluny III (avant-nef), à Saint-Leu-d'Esserent (avant-nef) et à Saint-Martindes-Champs, à Saint-Martin d'Avallon (chevet) et ailleurs... quasi simultanément ». À la suite d'Eduardo Carrero Santamaria ${ }^{45}$, on préférera alors inviter à considérer chaque édifice comme un unicum à étudier dans sa globalité et pas seulement pour sa place dans une chronologie bancale et obsolète. Cette démarche ne peut se faire qu'avec suffisamment de recul sur la nature profondément subjective de la recherche en tout temps : c'est à condition de prendre conscience de la partialité de ces débats qu'il sera possible de se réconcilier tout à fait avec une historiographie riche et de poursuivre le travail. Enfin, la question de la transmission se pose nécessairement: Thomas Flum souligne à raison la dualité du discours actuel, multiples nuances de la recherche d'une 
part et périodisations obsolètes et académiques de l'autre ${ }^{46}$. Certes, le temps de l'université et le volume horaire réduit des cours ne permettent pas d'approcher chaque période avec la subtilité qu'elle exigerait, mais la persistance évidente des lieux communs doit conduire à plus de précisions et de nuances dans le contenu délivré aux étudiants et futurs chercheurs.

11 À ces différentes remarques portées par l'ouvrage, on ajoutera également la nécessité de sortir de l'hyper-focalisation de la bibliographie sur les mêmes édifices ; Chartres, lieu d'accueil du colloque en 2015, en est un parfait exemple, tout comme un petit groupe d'églises, pour la plupart cathédrales (Paris, Bourges). Si l'on trouve aujourd'hui de salutaires études qui permettent de relativiser l'importance de certains d'entre eux durant tout le Moyen Âge, tant par le choix de leur architecture ou par l'aura de leur clergé $^{47}$, nous gagnerions tous à voir au-delà et à prendre, ou reprendre, l'étude d'édifices délaissés par l'historiographie. Mais «la critique est aisée et l'art est difficile » rappelait Philippe Néricault ; à ce titre, nous ne saurions nous éloigner de la science des auteurs qui nous ont précédés et qui nous entourent. Pour reprendre les mots de Bruno Phalip, il n'est pas nécessaire « d'être d'accord sur tous les points mais [...] de contribuer à promouvoir l'essor d'un débat bienveillant $»^{48}$.

Au-delà d'une présentation de l'état actuel de la recherche à un instant $\mathrm{T}$, ces deux ouvrages ont eu pour ambition, chacun à leur manière, de considérer leur renouvellement de leur discipline comme un objet d'étude à part entière. Dans le cas du premier, cette démarche a pris le sens d'un positionnement conceptuel, permettant de sortir la microarchitecture de l'association constante avec les seuls arts précieux et de montrer que celle-ci ne peut se définir comme une simple version miniature du grand. Dès lors, les questions d'échelle, tant en termes sociaux qu'en termes de création, s'effacent au profit d'autres problématiques plus stimulantes. L'objectif du second porte sur l'avenir de la recherche sur l'architecture gothique qui ne saurait être envisagé qu'à la condition de prendre conscience de l'héritage intellectuel des années et des siècles précédents. Au cœur de ces deux entreprises, la réévaluation du regard porté sur l'objet d'étude a permis sans nul doute d'écarter une partie des poncifs et d'évoquer de nouvelles et bénéfiques perspectives de recherche. Aucune d'entre elles n'a eu pour but de renier l'historiographie qui a pu construire ces disciplines, mais bien au contraire de s'en nourrir pour mieux en poursuivre l'analyse demain.

Reçu : 29 mars 2019 - Accepté : 21 mai 2020

\section{NOTES}

1. F. BUCHER, « Micro-Architecture as the "Idea" of Gothic Theory and Style », Gesta, 15/1-2 (1976), p. 71-89. 
2. Voir à ce sujet: https://www.inha.fr/fr/recherche/le-departement-des-etudes-et-de-larecherche/domaines-de-recherche/histoire-de-l-art-du-ive-au-xve-siecle/l-architecturehabitee-au-moyen-age-1.html (consulté le 28 mars 2019).

3. Voir à ce sujet: http://www.msh.univ-nantes.fr/08135357/0/fiche__article/ (consulté le 28 mars 2019).

4. Voir plus particulièrement C. KRATZKE et U. A LBRECHT, «Was ist Mikroarchitektur? Fragestellungen und Bandbreite der Erscheinungsformen », in C. KRATZKE et U. ALBRECHT (dir.), Mikroarchitektur im Mittelalter: ein gattungsübergreifendes Phänomen zwischen Realität und imagination, Leipzig, 2008.

5. F. BUCHER, « Micro-Architecture... », op. cit.

6. J.-M. GUILLOUËT, « Microarchitectures médiévales. Une perspective historiographique », p. 25.

7. F. BUCHER, "'Microarchitecture' as the Idea Of Gothic Theory and Style », in España entre el Mediterraneo y el Atlantico, Grenade, 1978, p. 487-496.

8. J.-M. GUILloUËT, « Microarchitectures médiévales... », op. cit., p. 25.

9. Parmi d'autres publications: A.T IMMERMANN, «Architectural Vision in Albrecht von Scharfenberg's Jüngerer Titurel. A vision of Architecture ?", in G. CLARKE et P. CROSSLEY (dir.), Architecture and Language. Constructing Identity in European Architecture c. 1000-c.1650, Cambridge, 2000, p. 58-71. Voir également la définition de la microarchitecture donnée par Achim Timmermann sur le site Oxfordartonline: http://www.oxfordartonline.com/groveart/view/ 10.1093/gao/9781884446054.001.0001/oao-9781884446054-e-7002216986?

_start=1\&pos=1\&q=microarchitecture\&search=quick (consulté le 28 mars 2019).

10. Notamment P. KURMANN, «Miniaturkathedrale oder monumentales Reliquiar? Zur architektur des Gertrudenschreines ", in V. HUCHARD et H. WESTERMANN-ANGERHAUSEN (dir.), Schatz aus den Trümmern. Der Silberschrein von Nivellles und die europaïsche Hochgotik, Cologne, 1995, p. 135-153.

11. E. M. KAVAlER, « Microarchitecture as the Paradigm of the Antique Architecture in the Low Countries : 1515-1540», p. 143-152.

12. J. GARDNER, «Who Were the Microarchitects? », p. 37-46.

13. M. J. SILLENCE, "Compositions and Associations of Architectural Frameworks on Cardinals' Seals in the Fifteenth Century », p. 101-107.

14. A. VILAIN et C. B LANC-RIEHL, "Le prince en architecture. Les sceaux du duc de Berry », p. 109-118.

15. A. PAOLICCHI, « Les chivote à l'époque de Constantin Brâncoveanu », p. 89-98.

16. F. TIXIER, «Dextérité de l'orfèvre, symbolisme de la forme. Autour de quelques crosses “architecturées" médiévales (XIV ${ }^{\mathrm{e}}-\mathrm{XVI}^{\mathrm{e}}$ siècles) », p. 119-127.

17. J. IBÁÑEZ F ERNÁNDEZ et A. Z ARAGOZÁ CATALÁN, «Microarchitecture in the Iberian Context between the Fourteenth and Sixteenth Centuries : A First Attempt at Analysis », p. 47-56.

18. A. TimmermanN, «Fleeting Glimpses of Eschaton: Scalar Travels in Medieval Microarchitecture », p. 57-67.

19. A. Collins, "Miniaturizing Mary: The Microarchitecture of Embodiment in the Sherborne Missal », p. 175-185.

20. A.-O. PoILPRÉ, «Bâtir et figurer la royauté chrétienne au IX siècle : les trônes architecturés des manuscrits de Charles le Chauve », p. 131-141.

21. F. MAKKI, «Figures scripturales de la microarchitecture aux palais de l'Alhambra de Grenade », p. 71-79.

22. F. ThÉNARD-DUVIVIER, Images sculptées au seuil des cathédrales. Les portails de Rouen, Lyon et Avignon (XIII -XIV siècles), Mont-Saint-Aignan, 2012.

23. S. BERGER, «Édifices miniaturisés et figures de bienfaiteurs à la période médiévale. Iconologie de la maquette d'architecture », p. 81-87. 
24. S. CLOART-PAWLAK, «Identification, hiérarchisation et sacralisation des espaces au seuil de l'église. Remarques sur le rôle des décors d'architecture à travers l'exemple de l'ornementation des portails gothiques », p. 165-173.

25. A. TIMBERT (dir.), Qu'est-ce que l'architecture gothique ? Essais, Villeneuve-d'Ascq, 2018.

26. A. TIMBERT (dir.), Architecture et sculpture gothiques. Renouvellement des méthodes et des regards, Rennes, 2012.

27. Dans une même perspective, plusieurs auteurs de l'ouvrage de 2018 (voir note 25) ont invité à d'autres occasions à une réflexion d'ensemble sur les méthodes de l'histoire de l'art médiéval au $\mathrm{XXI}^{\mathrm{e}}$ siècle, entre autres : A. TIMBERT, «Étudier l'architecture médiévale en France au tournant des $\mathrm{xx}^{\mathrm{e}}$ et $\mathrm{xxI}^{\mathrm{e}}$ siècles. Approche historiographique, propos méthodologique ", Hortus Artium medievalium, 25/1 (2019), p. 129-136; X. BARRAL I ALTET, «L'histoire de l'art médiéval à l'aube du $\mathrm{XXI}^{\mathrm{e}}$ siècle : des difficultés pour renouveler la discipline à un plaidoyer pour l'historiographie ", p. 14-19.

28. A. TIMBERT, « Introduction. Les voies et les mots d'une réponse », p. 13-27.

29. X. BARRAL I ALTET, «La cathédrale de Chartres, exemple emblématique des incertitudes de la discipline : positions théoriques, impasses idéologiques, questions ouvertes », p. 29-51.

30. Rappelons ici que l'adjectif apparaît pour la première fois dans plusieurs ouvrages italiens de la seconde moitié $\mathrm{du} \mathrm{Xv}^{\mathrm{e}}$ siècle, mais surtout dans la lettre de Raphaël au pape Léon $\mathrm{X}$, rédigée probablement dans l'année 1519, dans lequel celui-ci compare les édifices antiques aux édifices construits par les allemands - " gothiques ».

31. A. TIMBERT, «Introduction. Les voies et les mots d'une réponse », p. 13-27.

32. N. REVEYron, « De quoi le gothique est-il le nom ?», p. 91-107.

33. Voir également N. R EVEYRON, "Chronologie, périodisation, polychronie. Les temps de l'histoire de l'art médiévale », Perspectives, 4 (2008), p. 703-714.

34. T. FLUM, « Le gothique tardif et la visibilité des époques », p. 53-68.

35. C. FREIGANG, « Préface », p. 9-12.

36. L. GRANT, «Qu'est-ce que l'architecture gothique ? La perspective des mécènes », p. 129-143.

37. S. MURRAY, « Le grand dessein gothique », p. 109-128.

38. E. C ARRERO S ANTAMARIA, "L'étude de l'architecture gothique dans les cathédrales des royaumes de Castille et de León aux XII ${ }^{\mathrm{e}}$ et XIII ${ }^{\mathrm{e}}$ siècles. Une crise des modèles ", p. 69-90.

39. K. BENEŠOVSKÁ, «Ce qu'il advint de l'architecture gothique à la cour de Charles IV et de Venceslas IV », p. 181-209.

40. N. MASATSUGU, « Le gothique visible, le gothique invisible », p. 211-233.

41. L. GRANT, «Qu'est-ce que l'architecture... », op. cit.

42. P. CECCARINI, "Un modèle expérimental: la cathédrale gothique comme génétique de l'univers ", p. 145-179.

43. A. TIMBERT, «La cathédrale de Sens et l'architecture du $\mathrm{XII}^{\mathrm{e}}$ siècle. Une réflexion sur la méthode ", in J.-L. DAuphin, L. SAUlNiER-PERnuit et C. Pernuit-Farou (dir.), Saint-Étienne de Sens. La première cathédrale gothique dans son contexte, Sens, 2017, p. 9-15.

44. C. Pernuit, « Une "relecture" de la cathédrale de Sens (1130-1550) », Bulletin du Centre d'études médiévales d'Auxerre, 20/1 (2016), en ligne [https://journals.openedition.org/cem/14424].

45. E. CARRero SANTAMARIA, «L'étude de l'architecture gothique... », op. cit.

46. T. FLUM, « Le gothique tardif et la visibilité des époques », p. 53-68.

47. Entre autres, N. BALZAMO, "La renommée de Chartres au Moyen Âge », Bulletin de la Société archéologique d'Eure-et-Loir, 104 (2010), p. 1-14.

48. B. PHALIP, « Postface. De nouvelles terres gothiques ou l'expérience du pluriel », p. 233-242. 
INDEX

Mots-clés : microarchitecture, architecture gothique

\section{AUTEUR}

\section{CLAIRE PERNUIT}

Docteure en Histoire de l'art (Université de Bourgogne-Franche-Comté, Université de Paris Ouest Nanterre La Défense) 